\title{
An approximation formula in Hilbert space *
}

\author{
Zhihua Zhang and Naoki Saito \\ Dept. of Math., Univ. of California, Davis, California, 95616, USA. \\ E-mail: zzh@ucdavis.edu and saito@math.ucdavis.edu
}

\begin{abstract}
Let $\left\{\varphi_{k}\right\}_{1}^{\infty}$ be a frame for Hilbert space $H$. The purpose of this paper is to present an approximation formula of any $f \in H$ by a linear combination of finitely many frame elements in the frame $\left\{\varphi_{k}\right\}_{1}^{\infty}$ and show that the obtained approximation error depends on the bounds of frame and the convergence rate of frame coefficients of $f$ as well as the relation among frame elements.
\end{abstract}

Key words: approximation formula; linear combination; frame element; Hilbert space

MSC $42 \mathrm{C} 15$

\section{Introduction}

Let $H$ be a Hilbert space and $\left\{e_{k}\right\}_{1}^{\infty}$ be an orthonormal basis for $H$. It is well-known that any $f \in H$ can be approximated by the linear combination of $\left\{e_{k}\right\}_{1}^{n}$ and the approximation error depends on the convergence rate of the Fourier coefficients[2].

As a generalization of the orthonormal bases, Duffin and Schaeffer[3] introduced the notion of frames. Suppose that $\left\{\varphi_{k}\right\}_{1}^{\infty}$ is a frame for $H$. For any $f \in H$, we will construct a linear combination of finitely many frame elements in the frame $\left\{\varphi_{k}\right\}_{1}^{\infty}$ to approximate to $f$ and show that the approximation error depends on the bounds of frame and the convergence rate of frame coefficients of $f$ as well as the relation among frame elements.

We recall some concepts and propositions.

\footnotetext{
${ }^{*}$ The work was supported by NSF grant DMS-0410406 and Prof. Xiaoping Shen's NSF grant
} 
Let $\left\{\varphi_{k}\right\}_{1}^{\infty}$ be a sequence in Hilbert space $H$. If there exist two positive constants $A, B$ such that

$$
A\|f\|^{2} \leq \sum_{k=1}^{\infty}\left|\left(f, \varphi_{k}\right)\right|^{2} \leq B\|f\|^{2} \quad \forall f \in H
$$

then the sequence $\left\{\varphi_{k}\right\}_{1}^{\infty}$ is said to be a frame for $H$, where $A$ and $B$ are said to be frame bounds. Specially, if $A=B=1$ and $\left\|\varphi_{k}\right\|=1\left(k \in Z^{+}\right)$, then $\left\{\varphi_{k}\right\}_{1}^{\infty}$ is an orthonormal basis for $H$.

Let $\left\{\varphi_{k}\right\}_{1}^{\infty}$ be a frame for $H$. The frame operator $S$ is defined as

$$
S: \quad H \rightarrow H, \quad S f=\sum_{k=1}^{\infty}\left(f, \varphi_{k}\right) \varphi_{k} \quad \forall f \in H
$$

where $\left(f, \varphi_{k}\right)\left(k \in Z^{+}\right)$are said to be frame coefficients.

Proposition 1.1[1,p58-62]. Let $\left\{\varphi_{k}\right\}_{1}^{\infty}$ be a frame with bounds $A$ and $B$ for $H$. Then

(i) The frame operator $S$ is a self-conjugate operator and $\quad A\|f\| \leq\|S f\| \leq B\|f\| \quad \forall f \in H$.

(ii) The inverse operator $S^{-1}$ exists and $\quad \frac{1}{B}\|f\| \leq\left\|S^{-1} f\right\| \leq \frac{1}{A}\|f\| \quad \forall f \in H$.

(iii) Denote $\widetilde{\varphi}_{k}=S^{-1} \varphi_{k}$, the $\left\{\widetilde{\varphi}_{k}\right\}_{1}^{\infty}$ is also a frame for $H$ and

$$
\frac{1}{B}\|f\|^{2} \leq \sum_{k=1}^{\infty}\left|\left(f, \widetilde{\varphi}_{k}\right)\right|^{2} \leq \frac{1}{A}\|f\|^{2} \quad \forall f \in H
$$

(iv) For each $k$,

$$
\widetilde{\varphi}_{k}=\frac{2}{A+B} \varphi_{k}+\frac{2}{A+B} \sum_{n=1}^{\infty}\left(I-\frac{2 S}{A+B}\right)^{n} \varphi_{k}
$$

where $I$ is the identity operator.

(v) Let $R=I-\frac{2 S}{A+B}$. Then $\|R\| \leq \frac{B-A}{B+A}$.

The frame $\left\{\widetilde{\varphi}_{k}\right\}_{1}^{\infty}$ is said to be a dual frame of $\left\{\varphi_{k}\right\}_{1}^{\infty}$.

Denote the partial sum of the series in (1.4) by $\widetilde{\varphi}_{k}^{N}$, i.e.

$$
\widetilde{\varphi}_{k}^{0}=\frac{2}{A+B} \varphi_{k}, \quad \widetilde{\varphi}_{k}^{N}=\frac{2}{A+B} \varphi_{k}+\frac{2}{A+B} \sum_{n=1}^{N}\left(I-\frac{2 S}{A+B}\right)^{n} \varphi_{k}
$$

Proposition 1.2[1,p58-62]. Under the conditions of Proposition 1.1, then

(i) for any $f \in H$, the reconstruction formula $f=\sum_{k=1}^{\infty}\left(f, \varphi_{k}\right) \widetilde{\varphi}_{k}$ holds 
(ii) for any $f \in H$, the series $\sum_{k=1}^{\infty}\left(f, \varphi_{k}\right) \widetilde{\varphi}_{k}^{N}$ is convergent and

$$
\left\|f-\sum_{k=1}^{\infty}\left(f, \varphi_{k}\right) \widetilde{\varphi}_{k}^{N}\right\| \leq q^{N+1}\|f\| \quad\left(N \in Z^{+}\right)
$$

where $q=\frac{B-A}{B+A}$.

\section{A new frame approximation operator $\left(\sigma_{n}^{N}(\cdot)\right)_{m}$}

In order to approximate to any $f \in H$ by a linear combination of finitely many frame elements, we present a new frame approximation operator in this section.

Let $\left\{\varphi_{k}\right\}_{1}^{\infty}$ be a frame for $H$ with bounds $A$ and $B$. The frame operator $S, \widetilde{\varphi}_{k}$, and $\widetilde{\varphi}_{k}^{N}$ are stated in (1.2), (1.4), and (1.5), respectively.

Definition 2.1 We truncate the series $\sum_{k=1}^{\infty}\left(f, \varphi_{k}\right) \widetilde{\varphi}_{k}^{N}$ by its partial sum, for $n, N \in Z^{+}$, define

$$
\sigma_{n}^{N}(f):=\sum_{k=1}^{n}\left(f, \varphi_{k}\right) \widetilde{\varphi}_{k}^{N} \quad \forall f \in H
$$

Denote

$$
S_{m}: \quad H \rightarrow H, \quad S_{m} f:=\sum_{j=1}^{m}\left(f, \varphi_{j}\right) \varphi_{j} \quad \forall f \in H
$$

and

$$
\begin{array}{lll}
S_{m}^{1}:=S_{m}, & S_{m}^{l}:=S_{m}^{l-1}\left(S_{m}\right) & \left(l \in Z^{+}\right), \\
S^{1}:=S, & S^{l}:=S^{l-1}(S) & \left(l \in Z^{+}\right) .
\end{array}
$$

Definition 2.2. For any $k, N, m \in Z^{+}$, define

$$
\left(\widetilde{\varphi}_{k}^{0}\right)_{m}=\frac{2}{A+B} \varphi_{k} \quad \text { and } \quad\left(\widetilde{\varphi}_{k}^{N}\right)_{m}=\frac{2}{A+B} \varphi_{k}+\frac{2}{A+B} \sum_{n=1}^{N}\left(I-\frac{2 S_{m}}{A+B}\right)^{n} \varphi_{k} .
$$

From this definition, we have

$$
\left(\widetilde{\varphi}_{k}^{N}\right)_{m}=\left(\widetilde{\varphi}_{k}^{N-1}\right)_{m}+\frac{2}{A+B}\left(I-\frac{2 S_{m}}{A+B}\right)^{N} \varphi_{k} \quad\left(N \in Z^{+}\right) .
$$

Definition 2.3. Define a frame approximation operator as follows.

$$
\left(\sigma_{n}^{N}(\cdot)\right)_{m}: \quad H \rightarrow H, \quad\left(\sigma_{n}^{N}(f)\right)_{m}=\sum_{k=1}^{n}\left(f, \varphi_{k}\right)\left(\widetilde{\varphi}_{k}^{N}\right)_{m} \quad \forall f \in H .
$$


Lemma 2.4. (i) The sequence of operators $\left\{S_{m}\right\}_{1}^{\infty}$ is uniformly bounded.

(ii) For any $f \in H$,

$$
S_{m}^{l} f \rightarrow S^{l} f(m \rightarrow \infty) \quad \forall l \in Z^{+}
$$

Proof. Combining (1.2) with (2.2), for any $f \in H$, we have

$$
S_{m} f \rightarrow S f \quad(m \rightarrow \infty)
$$

Using the resonance theorem, we know that there exists $M>0$ such that $\left\|S_{m}\right\| \leq M\left(m \in Z^{+}\right)$. So we get (i).

We have known that (2.5) holds for $l=1$. Now we assume that (2.5) holds for $l-1$.

Noticing that $S_{m}^{l} f-S^{l} f=S_{m}\left(S_{m}^{l-1} f\right)-S_{m}\left(S^{l-1} f\right)+S_{m}\left(S^{l-1} f\right)-S\left(S^{l-1} f\right)$, we have

$$
\left\|S_{m}^{l} f-S^{l} f\right\| \leq\left\|S_{m}\left(S_{m}^{l-1} f-S^{l-1} f\right)\right\|+\left\|S_{m}\left(S^{l-1} f\right)-S\left(S^{l-1} f\right)\right\|=: p_{m}^{(l)}(f)+q_{m}^{(l)}(f)
$$

Since $\left\|S_{m}\right\| \leq M$, by the postulate of induction, we have

$$
p_{m}^{(l)}(f) \leq M\left\|S_{m}^{l-1} f-S^{l-1} f\right\| \rightarrow 0 \quad(m \rightarrow \infty)
$$

Let $g=S^{l-1} f$. Then by (2.6),

$$
q_{m}^{(l)}(f)=\left\|S_{m} g-S g\right\| \rightarrow 0(m \rightarrow \infty)
$$

Hence, by (2.7), we have $S_{m}^{l} f \rightarrow S^{l} f(m \rightarrow \infty)$, i.e. (2.5) holds for any $l \in Z^{+}$. So we get (ii).

Lemma 2.5. For any $f \in H,\left(\sigma_{n}^{N}(f)\right)_{m}$ is a linear combination of $\left\{\varphi_{j}\right\}_{1}^{\lambda}$, where $\lambda=\max \{m, n\}$.

Proof. By Definition 2.2 and the operator equality

$$
\left(I-\frac{2 S_{m}}{A+B}\right)^{n}=I+\sum_{l=1}^{n}\left(\begin{array}{c}
n \\
l
\end{array}\right)\left(-\frac{2}{A+B}\right)^{l} S_{m}^{l}
$$

we conclude that

$$
\left(\widetilde{\varphi}_{k}^{N}\right)_{m}=\frac{2(N+1)}{A+B} \varphi_{k}+\frac{2}{A+B} \sum_{n=1}^{N} \sum_{l=1}^{n}\left(\begin{array}{c}
n \\
l
\end{array}\right)\left(-\frac{2}{A+B}\right)^{l} S_{m}^{l} \varphi_{k} .
$$

Again by Definition 2.3, we get

$$
\left(\sigma_{n}^{N}(f)\right)_{m}=\frac{2(N+1)}{A+B} \sum_{k=1}^{n}\left(f, \varphi_{k}\right) \varphi_{k}+\frac{2}{A+B} \sum_{n=1}^{N} \sum_{l, k=1}^{n} b_{n, l, k} S_{m}^{l} \varphi_{k}=: M_{1}+M_{2},
$$


where $b_{n, l, k}=\left(\begin{array}{c}n \\ l\end{array}\right)\left(-\frac{2}{A+B}\right)^{l}\left(f, \varphi_{k}\right)$.

For any $f \in H$, by (2.2), we obtain that for any $k, l \in Z^{+}$,

$$
S_{m}^{l} \varphi_{k}=\sum_{j=1}^{m} c_{j} \varphi_{j}, \quad \text { where } \quad c_{j}=\sum_{\nu_{1}, \ldots, \nu_{l-1}=1}^{m}\left(\varphi_{k}, \varphi_{\nu_{1}}\right)\left(\varphi_{\nu_{1}}, \varphi_{\nu_{2}}\right) \cdots\left(\varphi_{\nu_{l-1}}, \varphi_{j}\right) .
$$

So we see that for $l, k \in Z^{+}, S_{m}^{l} \varphi_{k}$ is a linear combination of $m$ frame elements $\varphi_{1}, \varphi_{2}, \ldots, \varphi_{m}$, further, the sum $M_{2}$ is a linear combination of $m$ elements $\varphi_{1}, \varphi_{2}, \ldots, \varphi_{m}$. Clearly, the sum $M_{1}$ is a linear combination of $n$ elements $\varphi_{1}, \varphi_{2}, \ldots, \varphi_{n}$. Therefore, $\left(\sigma_{n}^{N}(f)\right)_{m}$ is a linear combination of $\left\{\varphi_{j}\right\}_{1}^{\lambda}$, where $\lambda=\max \{m, n\}$. Lemma 2.5 is proved.

\section{Approximation by $\left(\sigma_{n}^{N}(\cdot)\right)_{m}$}

We will approximate to $f$ by $\left(\sigma_{n}^{N}(f)\right)_{m}$. First, we estimate $\left\|f-\sigma_{n}^{N}(f)\right\|$ in Lemma 3.1. Next, we estimate $\left\|\sigma_{n}^{N}(f)-\left(\sigma_{n}^{N}(f)\right)_{m}\right\|$ in Lemma 3.3. Finally, we get an estimate $\left\|f-\left(\sigma_{n}^{N}(f)\right)_{m}\right\|$ in Theorem 3.4. Meanwhile, we show that the approximation error only depends on the frame bounds and the convergence rate of the frame coefficients of $f$ as well as the relation among frame elements.

Lemma 3.1. Let $\left\{\varphi_{k}\right\}_{1}^{\infty}$ be a frame for $H$ with bounds $A, B$ and $\sigma_{n}^{N}(f)$ be stated in (2.1). Denote

$$
\varepsilon_{n}(f):=\frac{1}{\|f\|}\left(\sum_{j=n+1}^{\infty}\left|\left(f, \varphi_{j}\right)\right|^{2}\right)^{\frac{1}{2}} .
$$

Then for any $f \in H$, we have

$$
\left\|f-\sigma_{n}^{N}(f)\right\| \leq q^{N+1}\|f\|+\frac{1}{\sqrt{A}}\left(1+q^{N+1}\right)\|f\| \varepsilon_{n}(f) \quad\left(n, N \in Z^{+}\right)
$$

where $q=\frac{B-A}{B+A}$.

Remark 3.2. Since $\left\{\varphi_{k}\right\}_{1}^{\infty}$ is a frame, we see that $\sum_{k=1}^{\infty}\left|\left(f, \varphi_{k}\right)\right|^{2}<\infty$. From this and (3.1), we get $\varepsilon_{n}(f) \rightarrow 0(n \rightarrow \infty)$

Proof of Lemma 3.1. By Proposition 1.2(ii) and (2.1), we know that for any $f \in H$ and $N \in Z^{+}$, the series $\sum_{k=1}^{\infty}\left(f, \varphi_{k}\right) \widetilde{\varphi}_{k}^{N}$ converges and $\sigma_{n}^{N}(f)=\sum_{k=1}^{n}\left(f, \varphi_{k}\right) \widetilde{\varphi}_{k}^{N}$ is its partial sum. Denote its remainder term by

$$
r_{n}^{N}(f):=\sum_{k=n+1}^{\infty}\left(f, \varphi_{k}\right) \widetilde{\varphi}_{k}^{N}
$$


So

$$
\left\|f-\sigma_{n}^{N}(f)\right\|=\left\|f-\left(\sum_{k=1}^{\infty}\left(f, \varphi_{k}\right) \widetilde{\varphi}_{k}^{N}-r_{n}^{N}(f)\right)\right\| \leq\left\|f-\sum_{k=1}^{\infty}\left(f, \varphi_{k}\right) \widetilde{\varphi}_{k}^{N}\right\|+\left\|r_{n}^{N}(f)\right\|
$$

Using Proposition 1.2(ii), we get

$$
\left\|f-\sigma_{n}^{N}(f)\right\| \leq q^{N+1}\|f\|+\left\|r_{n}^{N}(f)\right\| .
$$

By Proposition 1.2(i), the series $\sum_{k=1}^{\infty}\left(f, \varphi_{k}\right) \widetilde{\varphi}_{k}$ converges, so we can decompose $r_{n}^{N}(f)$ as follows:

$$
r_{n}^{N}(f)=\sum_{k=n+1}^{\infty}\left(f, \varphi_{k}\right) \widetilde{\varphi}_{k}+\sum_{k=n+1}^{\infty}\left(f, \varphi_{k}\right)\left(\widetilde{\varphi}_{k}^{N}-\widetilde{\varphi}_{k}\right)=: u_{n}^{N}(f)+v_{n}^{N}(f)
$$

By (1.4) and (1.5), it follows that

$$
\begin{aligned}
\widetilde{\varphi}_{k}-\widetilde{\varphi}_{k}^{N} & =\frac{2}{A+B} \sum_{n=N+1}^{\infty}\left(I-\frac{2 S}{A+B}\right)^{n} \varphi_{k} \\
& =\left(I-\frac{2 S}{A+B}\right)^{N+1}\left(\frac{2}{A+B} \varphi_{k}+\frac{2}{A+B} \sum_{n=1}^{\infty}\left(I-\frac{2 S}{A+B}\right)^{n} \varphi_{k}\right) \\
& =\left(I-\frac{2 S}{A+B}\right)^{N+1} \widetilde{\varphi}_{k}=R^{N+1} \widetilde{\varphi}_{k},
\end{aligned}
$$

where $R=I-\frac{2 S}{A+B}$. So we get

$$
v_{n}^{N}(f)=-\sum_{k=n+1}^{\infty}\left(f, \varphi_{k}\right) R^{N+1} \widetilde{\varphi}_{k}=-R^{N+1}\left(\sum_{k=n+1}^{\infty}\left(f, \varphi_{k}\right) \widetilde{\varphi}_{k}\right) .
$$

From this and (3.4), we get

$$
r_{n}^{N}(f)=\sum_{k=n+1}^{\infty}\left(f, \varphi_{k}\right) \widetilde{\varphi}_{k}-R^{N+1}\left(\sum_{k=n+1}^{\infty}\left(f, \varphi_{k}\right) \widetilde{\varphi}_{k}\right)=\left(I-R^{N+1}\right) \sum_{k=n+1}^{\infty}\left(f, \varphi_{k}\right) \widetilde{\varphi}_{k}
$$

However, we have

$$
\left\|\sum_{k=n+1}^{\infty}\left(f, \varphi_{k}\right) \widetilde{\varphi}_{k}\right\|^{2}=\sup _{\|g\|=1}\left|\left(\sum_{k=n+1}^{\infty}\left(f, \varphi_{k}\right) \widetilde{\varphi}_{k}, g\right)\right|^{2}=\sup _{\|g\|=1}\left|\sum_{k=n+1}^{\infty}\left(f, \varphi_{k}\right)\left(\widetilde{\varphi}_{k}, g\right)\right|^{2} .
$$

Using Cauchy's inequality in $l^{2}$, we get

$$
\left\|\sum_{k=n+1}^{\infty}\left(f, \varphi_{k}\right) \widetilde{\varphi}_{k}\right\|^{2} \leq\left(\sum_{k=n+1}^{\infty}\left|\left(f, \varphi_{k}\right)\right|^{2}\right) \cdot \sup _{\|g\|=1}\left(\sum_{k=n+1}^{\infty}\left|\left(\widetilde{\varphi}_{k}, g\right)\right|^{2}\right) .
$$


By Proposition 1.1 (iii), we know that $\left\{\widetilde{\varphi}_{k}\right\}_{1}^{\infty}$ is also a frame for $H$ and

$$
\sum_{k=1}^{\infty}\left|\left(\widetilde{\varphi}_{k}, g\right)\right|^{2} \leq \frac{1}{A}\|g\|^{2}
$$

From this, we get

$$
\left\|\sum_{k=n+1}^{\infty}\left(f, \varphi_{k}\right) \widetilde{\varphi}_{k}\right\|^{2} \leq \frac{1}{A}\left(\sum_{k=n+1}^{\infty}\left|\left(f, \varphi_{k}\right)\right|^{2}\right) .
$$

Again by (3.1) and (3.5), we have

$$
\left\|r_{n}^{N}(f)\right\| \leq\left\|I-R^{N+1}\right\| \cdot\left(\frac{1}{A}\|f\|^{2} \varepsilon_{n}^{2}(f)\right)^{\frac{1}{2}} .
$$

By Proposition 1.1(v), we have $\left\|I-R^{N+1}\right\| \leq 1+q^{N+1}\left(q=\frac{B-A}{B+A}\right)$. So

$$
\left\|r_{n}^{N}(f)\right\| \leq \frac{1}{\sqrt{A}}\left(1+q^{N+1}\right)\|f\| \varepsilon_{n}(f) .
$$

Finally, by (3.3), we obtain the conclusion of Lemma 3.1.

We will approximate to $\sigma_{n}^{N}(f)$ by $\left(\sigma_{n}^{N}(f)\right)_{m}$.

Lemma 3.3. Let $\left\{\varphi_{k}\right\}_{1}^{\infty}$ be a frame for $H$ with bounds $A, B$, and let $\sigma_{n}^{N}(f)$ and $\left(\sigma_{n}^{N}(f)\right)_{m}$ be stated in (2.1) and Definition 2.3, respectively. Then for any $f \in H$, we have

$$
\left\|\sigma_{n}^{N}(f)-\left(\sigma_{n}^{N}(f)\right)_{m}\right\| \leq \sqrt{B}\|f\|\left(1+\frac{2}{A+B}\right)^{N+1} \sqrt{n} \alpha_{N, n, m} \quad\left(N, n, m \in Z^{+}\right),
$$

where

$$
\alpha_{N, n, m}:=\max _{\substack{1 \leq l \leq N \\ 1 \leq k \leq n}}\left\|S^{l} \varphi_{k}-S_{m}^{l} \varphi_{k}\right\|
$$

Proof. By (1.5), we have

$$
\widetilde{\varphi}_{k}^{N}=\widetilde{\varphi}_{k}^{N-1}+\frac{2}{A+B} \varphi_{k}+\frac{2}{A+B} \sum_{l=1}^{N}\left(\begin{array}{c}
N \\
l
\end{array}\right)\left(-\frac{2}{A+B}\right)^{l} S^{l} \varphi_{k} .
$$

and by (2.4), we have

$$
\left(\widetilde{\varphi}_{k}^{N}\right)_{m}=\left(\widetilde{\varphi}_{k}^{N-1}\right)_{m}+\frac{2}{A+B} \varphi_{k}+\frac{2}{A+B} \sum_{l=1}^{N}\left(\begin{array}{c}
N \\
l
\end{array}\right)\left(-\frac{2}{A+B}\right)^{l} S_{m}^{l} \varphi_{k}
$$


So we get

$$
\widetilde{\varphi}_{k}^{N}-\left(\widetilde{\varphi}_{k}^{N}\right)_{m}=\widetilde{\varphi}_{k}^{N-1}-\left(\widetilde{\varphi}_{k}^{N-1}\right)_{m}+\frac{2}{A+B} \sum_{l=1}^{N}\left(\begin{array}{c}
N \\
l
\end{array}\right)\left(-\frac{2}{A+B}\right)^{l}\left(S^{l} \varphi_{k}-S_{m}^{l} \varphi_{k}\right) .
$$

Recursively using the above formula, we have

$$
\widetilde{\varphi}_{k}^{N}-\left(\widetilde{\varphi}_{k}^{N}\right)_{m}=\left(\widetilde{\varphi}_{k}^{1}-\left(\widetilde{\varphi}_{k}^{1}\right)_{m}+\frac{2}{A+B} \sum_{j=2}^{N} \sum_{l=1}^{j}\left(\begin{array}{c}
j \\
l
\end{array}\right)\left(-\frac{2}{A+B}\right)^{l}\left(S^{l} \varphi_{k}-S_{m}^{l} \varphi_{k}\right) .\right.
$$

By Definition 2.2 and (1.5), we get

$$
\widetilde{\varphi}_{k}^{1}-\left(\widetilde{\varphi}_{k}^{1}\right)_{m}=-\left(\frac{2}{A+B}\right)^{2}\left(S \varphi_{k}-S_{m} \varphi_{k}\right) .
$$

From this and (3.8), we have

$$
\widetilde{\varphi}_{k}^{N}-\left(\widetilde{\varphi}_{k}^{N}\right)_{m}=\frac{2}{A+B} \sum_{j=1}^{N} \sum_{l=1}^{j}\left(\begin{array}{c}
j \\
l
\end{array}\right)\left(-\frac{2}{A+B}\right)^{l}\left(S^{l} \varphi_{k}-S_{m}^{l} \varphi_{k}\right) .
$$

So we obtain

$$
\left\|\widetilde{\varphi}_{k}^{N}-\left(\widetilde{\varphi}_{k}^{N}\right)_{m}\right\| \leq \frac{2}{A+B}\left(\sum_{j=1}^{N} \sum_{l=1}^{j}\left(\begin{array}{c}
j \\
l
\end{array}\right)\left(\frac{2}{A+B}\right)^{l}\right) \cdot \max _{1 \leq l \leq N}\left\|S^{l} \varphi_{k}-S_{m}^{l} \varphi_{k}\right\| .
$$

However,

$$
\frac{2}{A+B} \sum_{j=1}^{N} \sum_{l=1}^{j}\left(\begin{array}{c}
j \\
l
\end{array}\right)\left(\frac{2}{A+B}\right)^{l} \leq \frac{2}{A+B} \sum_{j=1}^{N}\left(1+\frac{2}{A+B}\right)^{j} \leq\left(1+\frac{2}{A+B}\right)^{N+1}
$$

So we have

$$
\left\|\widetilde{\varphi}_{k}^{N}-\left(\widetilde{\varphi}_{k}^{N}\right)_{m}\right\| \leq\left(1+\frac{2}{A+B}\right)^{N+1} \cdot \max _{1 \leq l \leq N}\left\|S^{l} \varphi_{k}-S_{m}^{l} \varphi_{k}\right\| .
$$

By (2.1) and Definition 2.3, using cauchy's inequality, we obtain that for any $f \in H$,

$$
\begin{aligned}
\left\|\sigma_{n}^{N}(f)-\left(\sigma_{n}^{N}(f)\right)_{m}\right\| & \leq \sum_{k=1}^{n}\left|\left(f, \varphi_{k}\right)\right| \cdot\left\|\widetilde{\varphi}_{k}^{N}-\left(\widetilde{\varphi}_{k}^{N}\right)_{m}\right\| \\
& \leq\left(\sum_{k=1}^{n}\left|\left(f, \varphi_{k}\right)\right|^{2}\right)^{\frac{1}{2}} \cdot\left(\sum_{k=1}^{n}\left\|\widetilde{\varphi}_{k}^{N}-\left(\widetilde{\varphi}_{k}^{N}\right)_{m}\right\|^{2}\right)^{\frac{1}{2}}
\end{aligned}
$$

Combining (3.9)-(3.10) with (3.6), we get

$$
\left\|\sigma_{n}^{N}(f)-\left(\sigma_{n}^{N}(f)\right)_{m}\right\| \leq\left(1+\frac{2}{A+B}\right)^{N+1} \sqrt{n} \alpha_{N, n, m}\left(\sum_{k=1}^{n}\left|\left(f, \varphi_{k}\right)\right|^{2}\right)^{\frac{1}{2}}
$$


From this and (1.1), we get the conclusion of Lemma 3.3.

Since

$$
\left\|f-\left(\sigma_{n}^{N}(f)\right)_{m}\right\| \leq\left\|f-\sigma_{n}^{N}(f)\right\|+\left\|\sigma_{n}^{N}(f)-\left(\sigma_{n}^{N}(f)\right)_{m}\right\|,
$$

by Lemma 3.1 and Lemma 3.3, we conclude immediately the following

Theorem 3.4. Let $\left\{\varphi_{k}\right\}_{1}^{\infty}$ be a frame with bounds $A$ and $B$, and let

$$
\left(\sigma_{n}^{N}(f)\right)_{m}=\sum_{k=1}^{N}\left(f, \varphi_{k}\right)\left(\widetilde{\varphi}_{k}^{N}\right)_{m} \quad(f \in H)
$$

where $\left(\widetilde{\varphi}_{k}^{N}\right)_{m}$ is stated in Definition 2.2. Then for any $f \in H$ and $N, n, m \in Z^{+}$, we have

$$
\begin{aligned}
\left\|f-\left(\sigma_{n}^{N}(f)\right)_{m}\right\| & \leq q^{N+1}\|f\| \\
& +\frac{1}{\sqrt{A}}\left(1+q^{N+1}\right)\|f\| \varepsilon_{n}(f)+\sqrt{B}\|f\|\left(1+\frac{2}{A+B}\right)^{N+1} \sqrt{n} \alpha_{N, n, m},
\end{aligned}
$$

where $\varepsilon_{n}(f)$ is stated in $(3.1), q=\frac{B-A}{B+A}$, and

$$
\left.\alpha_{N, n, m}=\max _{\substack{1 \leq l \leq N \\ 1 \leq k \leq n}}\left\|S^{l} \varphi_{k}-S_{m}^{l} \varphi_{k}\right\| \quad \text { (S is the frame operator }\right) .
$$

Remark 3.5. In Lemma 2.5, we have shown that $\left(\sigma_{n}^{N}(f)\right)_{m}$ is a linear combination of $\varphi_{1}, \ldots, \varphi_{\lambda}(\lambda=$ $\max \{m, n\})$. So Theorem 3.4 gives a formula approximating to any $f \in H$ by a linear combination of finitely many frame elements.

Remark 3.6. Denote

$$
\begin{aligned}
R_{1} & :=q^{N+1}\|f\|, \\
R_{2} & :=\frac{1}{\sqrt{A}}\left(1+q^{N+1}\right)\|f\| \varepsilon_{n}(f), \\
R_{3} & :=\sqrt{B}\|f\|\left(1+\frac{2}{A+B}\right)^{N+1} \sqrt{n} \alpha_{N, n, m} .
\end{aligned}
$$

Since $q=\frac{B-A}{B+A}<1$, we see that $R_{1} \rightarrow 0(N \rightarrow \infty)$. By Remark 3.2, we have $R_{2} \rightarrow 0(n \rightarrow \infty)$. From Lemma 2.4(ii) and (3.6), we obtain $\alpha_{N, n, m} \rightarrow 0(m \rightarrow \infty)$, so we have $R_{3} \rightarrow 0(m \rightarrow \infty)$. 
Therefore, for any $f \in H$ and an approximation error $\varepsilon>0$, first we choose $N$ such that $R_{1}<\frac{\varepsilon}{3}$, next, we choose $n$ such that $R_{2}<\frac{\varepsilon}{3}$, finally, for fixed $N, n$, we choose $m$ such that $R_{3}<\frac{\varepsilon}{3}$. Then from (3.11), we have $\left\|f-\left(\sigma_{n}^{N}(f)\right)_{m}\right\|<\varepsilon$.

Our result is a generalization of a known result on the orthonormal bases[2].

Remark 3.7. When $\left\{\varphi_{k}\right\}$ is an orthonormal basis for $H$, the frame bounds $B=A=1$ and the frame operator $S$ and $S_{m}$ are both the identity operator. By (3.6),

$$
\alpha_{N, n, m}=0
$$

By (1.4), (1.5), (2.3), and (3.12), we have

$$
\widetilde{\varphi}_{k}=\widetilde{\varphi}_{k}^{N}=\left(\widetilde{\varphi}_{k}^{N}\right)_{m}=\varphi_{k} \quad \text { and } \quad R_{1}=R_{3}=0, \quad R_{2}=\left(\sum_{k=n+1}^{\infty}\left|\left(f, \varphi_{k}\right)\right|^{2}\right)^{\frac{1}{2}}
$$

Therefore, Theorem 3.4 is reduced to a well-known result in Hilbert space[2].

\section{References}

[1] Daubechies I., Ten lectures on wavelets, CBMS- Conference Lecture Notes, 6 (1992) SIAM, Philadelphia.

[2] DeVore, R.A., Nonlinear approximation, Acta. Numerica, (1998) 51-150.

[3] Duffin R.J. and Schaeffer A., A class of nonharmonic Fourier series, Trans. Amer. Math. Soc., 72 (1952) $341-366$. 